provisional, though.

Several other potential MS treatments are now being tested in MS patients. For instance, Biogen's (Cambridge, MA) version of beta interferon is in phase III trials. In addition, a copolymer that mimicks some activities of a protein associated with myelin is being clinically tested by Teva Pharmaceutical In- dustries (Jerusalem). And Autoimmune (Lexington, MA) is pushing AI-100, which is derived from myelin, through clinical trials.

—Jeffrey L. Fox

\title{
Centocor's CentoRx benefits risky angioplasty
}

NEW YORK-Over the past year Centocor's (Malvern, PA) sepsis product, Centoxin, has dealt the company one staggering blow after another, knocking an astronomical $\$ 1.4$ billion off the firm's market valuation. Most recently, Centocor scrapped its second phase III clinical trial of Centoxin-a monoclonal antibody (MAb) that targets sepsis caused by gram-negative bacteriabecause of excess deaths in Centoxin-treated patients without gram-negative bacteremia.

Yet recently reported, positive phase III results of a second Centocor product, CentoRx, could help get Centocor off the canvas. CentoRx is a chimeric MAb fragment that binds to the GPIIb/IIIa receptor on blood platelets, preventing platelets from clumping together to form blood clots. CentoRx's phase III trial targeted blood-clotrelated complications of high-risk coronary angioplasty. Angioplasty - which aims to expand narrowed coronary arteries to improve blood flow to the heart--uses a balloon-tipped catheter that is inserted into an artery in the leg and then maneuvered to the obstructed coronary artery, where the balloon is inflated. Yet the procedure sometimes injures the coronary arterial wall, prompting blood-clot formation and abrupt reclosing of the artery, despite the use of a standard anticlotting regimen of aspirin and heparin. Artery reclosing can cause such adverse complications as death, heart attack, or the need for emergency, repeat angioplasty. Angioplasty patients considered at high risk for these complications include those with unstable angina or with recent heart attacks.

\section{Phase III}

CentoRx's phase III trial involved 2,099 patients at 56 medical centers and was conducted by the Thrombolysis and Angioplasty in Myocardial Infarction Group, a clinical-trials organization dedicated to testing new cardiovascular compounds. The patients - all of whom received aspirin and heparin-were divided into three groups: those receiving placebo, those receiving an injection of CentoRx, and those receiving a CentoRx injection and a follow-up CentoRx infusion. The injection totaled 0.25 $\mathrm{mg} / \mathrm{kg}$ of CentoRx, while the infusion came to $7.2 \mathrm{mg}$ of CentoRx administered over 12 hours.

In the placebo group, 12.8 percent of patients suffered death, heart attack, or repeat angioplasty in the 30 days following their angioplasty. In the group receiving a CentoRx injection, 11.4 percent of patients suffered such complications, a statistically insignificant 10 percent reduction, compared to the placebo group. Of patients receiving a CentoRx injection and CentoRx infusion, 8.3 percent suffered complications, a statistically significant 35 percent drop, compared to placebo. "The inhibition of platelet aggregation must be sustained through a follow-up infusion to achieve a marked clinical effect," says a Centocor spokesman.

CentoRx's biggest side effect was increased bleeding, typically at the puncture site where the catheter entered the patient's leg. While 7 percent of placebo-treated patients suffered major bleeds - which were defined as a $5 \mathrm{gm} / \mathrm{dl}$ drop in hemoglobin - fully 14 percent of patients receiving CentoRx injections and infusions experienced such bleeds, and 11 percent of patients given CentoRx injections suffered such bleeds. Yet no differences occurred among treatment groups for more serious bleeding episodes, including intracranial bleeding and bleeding complications during emergency intervention.

\section{Lilly}

CentoRx's bleeding side effect could prove significant. Indeed, before granting CentoRx marketing approval, the Food and Drug Administration (FDA, Bethesda, MD) may ask for an additional trial to demonstrate better control of bleeding. "In any event, Centorx won't be widely used until Centocor can better show how to control bleeding, and this will require more follow-up trials," says David Ebersman, a biotech analyst at Oppenheimer (New York).

Centocor plans to submit a product license application for Cento $\mathrm{Rx}$ to FDA later this year. As part of a 1992 Centoxin licensing agreement, however, Eli Lilly (Indianapolis, IN) has the option to acquire distribution rights to CentoRx for $\$ 25$ million if Centoxin is approved this year or for no charge if Centoxin isn't approved this year. With the cancellation of Centoxin's latest phase III trial, Centoxin approval this year is nearly impossible, making it all the more feasible for Lilly to choose to distribute CentoRx. Centocor would then receive manufacturing revenues for CentoRx, which would total at least 50 percent of the product's retail price.

If approved for high-risk angioplasty, CentoRx could find use in 60,000 angioplasties a year in the U.S., or 20 percent of the 300,000 annual U.S. angioplasties. Analysts have pegged this market at up to $\$ 70$ million a year. CentoRx, moreover, has completed phase II studies in unstable angina and heart attack.

Among novel anticoagulants, CentoRx is perhaps the closest to market. Yet anticoagulant research is incredibly competitive, with platelet inhibitors "probably the most competitive area of anticoagulant research," says Oppenheimer's Ebersman, adding that "it seems that every large pharmaceutical company is committing some effort to developing an antagonist to the GPIIb/IIIa platelet receptor." Among these firms are Merck (Rahway, NJ), Hoffmann-La Roche (Nutley, NJ), Bristol-Myers Squibb (New York), SmithKline Beecham (Philadelphia, PA), Boehringer Ingelheim (Ridgefield, CT), and G.D. Searle (Skokie, IL). -B.J. Spalding
Centocor plans

to submit a

product license

application for

CentoRx to

FDA later this

year. Lilly,

however, has the

option to

distribute the

compound. 